\title{
Improving student understanding of Dirac notation by using analogical reasoning in the context of a three-dimensional vector space
}

\author{
Emily Marshman ${ }^{1}$ and Chandralekha Singh ${ }^{2}$ \\ ${ }^{I}$ Department of Physics, Community College of Allegheny County, Pittsburgh, PA, 15212 \\ ${ }^{2}$ Department of Physics and Astronomy, University of Pittsburgh, Pittsburgh, PA, 15260
}

\begin{abstract}
Dirac notation is a compact and elegant notation taught to students in advanced quantum mechanics courses. However, students struggle to master Dirac notation and have difficulty translating between the Dirac notation and representations of the same quantum mechanical entity in different situations. We discuss an investigation of student difficulties with Dirac notation in the context of a three-dimensional vector space and the development, validation and evaluation of a Quantum Interactive Learning Tutorial (QuILT) that uses analogical reasoning to improve student understanding of this notation. The QuILT uses analogical reasoning and builds on students' prior knowledge of three-dimensional vectors in the familiar context of introductory mechanics to help students build a coherent understanding of Dirac notation before transitioning to the quantum mechanical context. We summarize the development and validation of the QuILT and findings from the inclass evaluation.
\end{abstract}




\section{BACKGROUND AND GOAL}

While the role of quantum mechanics $(\mathrm{QM})$ in science and technology innovation has been consistent, learning QM is challenging even for advanced undergraduate and graduate students [1-23]. Investigations of student difficulties in learning QM are important for developing curricula and pedagogies to help students learn QM [1-23].

However, few prior research studies focus on difficulties with Dirac notation [6-9], a compact and convenient notation used extensively in upper-level QM. Dirac notation can be viewed as analogous in terms of its compactness and convenience to Feynman diagrams in quantum field theory, which are elegant and help simplify complicated multidimensional integrals representing various physical processes involving different particles. Moreover, similar to Feynman diagrams, Dirac notation is not familiar to students from their earlier courses so that mastering this useful notation poses great challenges for even advanced students.

Here we discuss an investigation of student difficulties with Dirac notation in the context of a three-dimensional vector space in undergraduate $\mathrm{QM}$ which was used as a guide for the development, validation and evaluation of a Quantum Interactive Learning Tutorial (QuILT) to improve student understanding of this notation. The QuILT strives to help students develop a good grasp of Dirac notation by using analogical reasoning, which has been researched extensively, e.g., see Refs. [24-37]. In physics, analogical reasoning has been found to be effective for helping introductory physics students transfer their learning from one context to another, e.g., see Refs. [24-31]. In the context of Dirac notation in three dimensions, the major learning goal of the QuILT is to help students develop facility with the notation and be able to translate between the Dirac notation and representations of the same quantum entity in different situations, e.g., matrix representations of the quantum states, the quantum mechanical operators, etc. in a given basis.

The QuILT strives to help students develop expertise and facility in using Dirac notation by making analogies between the standard vector notation learned in a three dimensional vector space, e.g., in introductory mechanics, and the Dirac notation. We hypothesized that students may be able to develop a good conceptual and procedural understanding of Dirac notation if they have opportunities to make analogies between the familiar vector notation and Dirac notation. In particular, we hypothesized that even though the surface features of the standard vector notation and Dirac notation are different (e.g., the vector notation uses arrows whereas Dirac notation uses bras and kets), students can use familiar concepts in the standard vector notation and learn to express them in Dirac notation. Then, once they are familiar with the Dirac notation in the familiar three dimensional context, they can make sense of the Dirac notation in the context of QM.

For example, in a three dimensional vector space, a force vector can be written as $\vec{F}=a \hat{\imath}+b \hat{\jmath}+c \hat{k}$ in the standard vector notation. The components of the force vector $\vec{F}$ along the orthonormal basis vectors $\hat{\imath}, \hat{\jmath}$ and $\hat{k}$ are the projections of $\vec{F}$ along the basis vectors $\hat{\imath}, \hat{\jmath}$ and $\hat{k}$, respectively (i.e., $a, b$, and $c$ ). To find the projection of $\vec{F}$ along the basis vector $\hat{\imath}$, one can find the scalar product $\hat{\imath} \cdot \vec{F}=\hat{\imath} \cdot(a \hat{\imath}+b \hat{\jmath}+c \hat{k})=$ $a$. In Dirac notation, the force vector $\vec{F}$ can be written as a ket vector $|F\rangle=a|i\rangle+b|j\rangle+c|k\rangle$. The components of the force vector $|F\rangle$ along the basis vectors can be found by projecting $|F\rangle$ along the basis vectors $|i\rangle,|j\rangle$, and $|k\rangle$. For example, the component of $|F\rangle$ along the basis vector $|i\rangle$ is found by projecting $|F\rangle$ along the basis vector $|i\rangle$, i.e., the scalar (inner) product of the bra vector $\langle i|$ with the ket vector $|F\rangle,\langle i \mid F\rangle=a$. The projection operator that projects $|F\rangle$ along the basis vector $|i\rangle$ is the outer product $|i\rangle\langle i|$. The projection operator for a particular basis vector acting on vector $|F\rangle$ gives the component of $|F\rangle$ along the basis vector times the same basis vector, i.e., $|i\rangle\langle i \mid F\rangle=a|i\rangle$. The sum of all of the projection operators corresponding to an orthonormal basis set gives the identity operator $\hat{I}$, e.g., $\hat{I}=$ $|i\rangle\langle i|+| j\rangle\langle j|+| k\rangle\langle k|$. The identity operator acting on a vector gives the same vector back, e.g., $\hat{I}|F\rangle=(|i\rangle\langle i|+| j\rangle\langle j|+$ $|k\rangle\langle k|)|F\rangle=a|i\rangle+b|j\rangle+c|k\rangle=|F\rangle$.

Once an orthonormal basis is chosen to represent vectors and operators, they can be represented as matrices in the given basis. In the matrix representation, ket vectors are represented as column matrices and bra vectors are represented as row matrices. If one chooses an orthonormal basis $\{|i\rangle,|j\rangle$, and $|k\rangle\}$ such that $|i\rangle \doteq\left(\begin{array}{l}1 \\ 0 \\ 0\end{array}\right),|j\rangle \doteq\left(\begin{array}{l}0 \\ 1 \\ 0\end{array}\right)$, and $|k\rangle \doteq$ $\left(\begin{array}{l}0 \\ 0 \\ 1\end{array}\right)$ (in which the symbol "=" means "is represented by" in the chosen basis), the vector $|F\rangle$ can be represented as a column vector (or matrix) as $|F\rangle \doteq a\left(\begin{array}{l}1 \\ 0 \\ 0\end{array}\right)+b\left(\begin{array}{l}0 \\ 1 \\ 0\end{array}\right)+c\left(\begin{array}{l}0 \\ 0 \\ 1\end{array}\right)=\left(\begin{array}{l}a \\ b \\ c\end{array}\right)$. The component of the force vector $|F\rangle$ along, e.g., basis vector $|i\rangle$, is found by projecting $|F\rangle$ along the basis vector $|i\rangle$, i.e., the scalar product of the bra vector $\langle i|$ with ket vector $|F\rangle,\langle i \mid F\rangle \doteq\left(\begin{array}{lll}1 & 0 & 0\end{array}\right)\left(\begin{array}{l}a \\ b \\ c\end{array}\right)=a$. The projection operator that projects $|F\rangle$ along the basis vector $|i\rangle$ is $|i\rangle\langle i|$ and can be represented as a matrix in the given basis as $|i\rangle\langle i| \doteq$

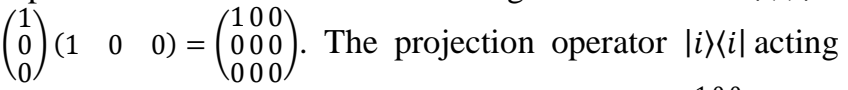

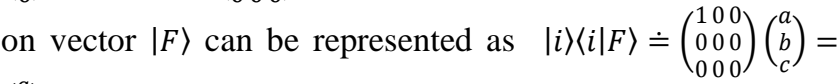
$\left(\begin{array}{l}a \\ 0 \\ 0\end{array}\right)=a|i\rangle$. The identity operator $\hat{I}$ can be represented by a square matrix in the given basis with ones along the diagonal

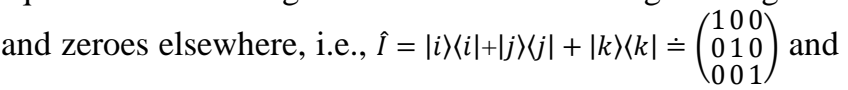
is the same in all representations (bases) chosen.

\section{METHODOLOGY AND OVERVIEW}

The development and validation of the QuILT and the corresponding pre/posttests started with an investigation of 
undergraduate student difficulties in a required upper-level undergraduate QM course for junior/senior physics majors at a large research university after traditional instruction in relevant concepts and by conducting a cognitive task analysis of the requisite knowledge [38]. The QuILT strives to help students build on their prior knowledge and addresses common difficulties found via research. After a preliminary version of the QuILT was developed based upon the cognitive task analysis of the underlying knowledge [38] from both expert and novice perspectives, it underwent many iterations between the two researchers and then was iterated several times with three physics faculty members (who teach advanced courses regularly) and two other graduate students who conduct research in physics education research to ensure that they agreed with the content and wording. It was also administered to students who were taking or had taken traditionally taught upper-level QM courses using individual think-aloud interviews to ensure that the guided approach was effective and the questions were unambiguously interpreted. The interviews also allowed us to better understand the rationale for student responses. During the semi-structured interviews, students were asked to "think aloud" while answering the questions. Students first read the questions on their own and answered them without interruptions except that they were prompted to think aloud if they were quiet for a long time. After students had finished answering a particular question to the best of their ability, they were asked to further clarify and elaborate on issues that they had not clearly addressed earlier. Modifications and improvements were made to the QuILT based upon the student and faculty feedback.

The QuILT uses a guided inquiry-based approach to learning and actively engages students in the learning process. It includes a pretest that included the questions shown in Table I after traditional instruction in relevant concepts. Instruction in class involved a review of how a vector written in the form $\vec{F}=a \hat{\imath}+b \hat{\jmath}+c \hat{k}$ can be written as $|F\rangle=a|i\rangle+b|j\rangle+c|k\rangle$ in Dirac notation. Students were also taught how to represent vectors (kets) as column matrices, bra vectors as row matrices, and operators as square matrices. The concepts of the identity operator and projection operator were also covered in class.

Students engaged with the tutorial in small groups in class (but could also work on it alone when using it as a self-paced learning tool in homework). As students worked through the tutorial, they are asked to predict what should happen in a given situation. Then, the tutorial strives to provide scaffolding and feedback as needed to bridge the gap between their initial knowledge and the level of understanding that is desired. Students are also provided checkpoints to reflect upon what they have learned and to make explicit the connections between what they are learning and their prior knowledge. They are given an opportunity to reconcile the differences between their predictions and the guidance provided in the checkpoints before proceeding further. The Dirac notation QuILT builds on students' prior knowledge of the standard vector notation using basis vectors $\hat{\imath}, \hat{\jmath}$, and $\hat{k}$. Students are guided to translate vectors written in the form $\vec{F}=a \hat{\imath}+b \hat{\jmath}+c \hat{k}$ to Dirac notation, e.g., $|F\rangle=a|i\rangle+b|j\rangle+c|k\rangle$. Analogies are drawn between scalar product notation and the inner product in Dirac notation, e.g., $\hat{\imath} \cdot \vec{F}$ and $\langle i \mid F\rangle$. Then, students are guided to represent the vector $|F\rangle$ in a particular basis, e.g., $|F\rangle \doteq\left(\begin{array}{l}a \\ b \\ c\end{array}\right)$ and learn to translate between different bases. Next, students learn that, in a given basis, ket states can be represented by column vectors, bra states can be represented by row vectors, inner products can be represented as numbers, and outer products are operators which can be represented as square matrices. Then, students are guided to develop a functional understanding of the spectral decomposition of the identity operator (or completeness relation) in the familiar context of a three-dimensional vector space. Students also determine the identity operator in a different orthonormal basis and verify that it is represented as the same matrix regardless of the basis chosen. Lastly, the QuILT strives to help students learn about a projection operator and that it returns a unit vector along the direction projected multiplied by a number (the component of a vector along the direction projected).

After working on the QuILT in class, the students were given one week to work through the rest of it as part of their homework assignment. Once the students turned in the QuILT as their homework assignment, the students were given an in-class posttest that had the same questions as the pretest (see Table I). The posttests were graded for correctness and counted as a quiz in the course.

After the development and validation of the QuILT and pre/posttests, it was administered to 57 students in three upper-level undergraduate QM courses. In the first year of administration, students were not given a pretest, so the number of students taking the pretest is lower $(N=43)$.

\section{EVALUATION OF THE QUILT}

Table II shows the average pretest and posttest scores and Table III shows the types of difficulties students had on the pretest and posttest. The open-ended questions on the pretests and posttests were graded using rubrics which were developed by two of the investigators together. A subset of the open-ended questions was graded separately by the investigators. After comparing the grading of the open-ended questions first, the investigators discussed any disagreements in grading and resolved them with a final inter-rater reliability of better than $95 \%$. Below, we discuss the difficulties commonly observed in individual interviews and on the pretest, and students' pretest and posttest performance on each question.

Difficulty with the components of a threedimensional vector in Dirac notation. Some students had difficulties writing the components of a three dimensional vector in Dirac notation. Table II shows that, on pretest 
Table I. Questions about Dirac notation in three-dimensional vector space given to upper-level undergraduate students. Q1 Assume that $|i\rangle,|j\rangle$, and $|k\rangle$ form a complete set of orthonormal basis vectors. For vector $\left|\chi_{1}\right\rangle=a|i\rangle+b|j\rangle+$ $c|k\rangle$ (a vector in a three-dimensional vector space):

a. write the components $\mathrm{a}, \mathrm{b}$, and $\mathrm{c}$ in Dirac notation.

b. represent $\left|\chi_{1}\right\rangle$ as a column vector in the given basis.

Q2 Assume that $|i\rangle,|j\rangle$, and $|k\rangle$ form a complete set of orthonormal basis vectors. $\left|\chi_{1}\right\rangle=a|i\rangle+b|j\rangle+c|k\rangle$ and $\left|\chi_{2}\right\rangle=d|i\rangle+$ $e|j\rangle+f|k\rangle$ are vectors in a three dimensional vector space.

a. Write the outer product of "ket" vector $\left|\chi_{1}\right\rangle$ with "bra" vector $\left\langle\chi_{2}\right|$ in the given basis.

b. Is this outer product a scalar (number), a column vector, a row vector, or a $3 \times 3$ matrix in the given basis?

Q3 Write the identity operator in terms of $|i\rangle,|j\rangle$, and $|k\rangle$, which form a complete set of orthonormal basis vectors for a threedimensional vector space.

Q4 For the vector $\left|\chi_{1}\right\rangle=a|i\rangle+b|j\rangle+c|k\rangle$ :

Write down the projection operator that projects vector $\left|\chi_{1}\right\rangle$ along the direction of the unit vector $|i\rangle$. Then, using that projection operator, show what happens to the vector $\left|\chi_{1}\right\rangle$ when the projection operator acts on it. Summarize your result in one sentence.

question Q1.a, only $49 \%$ of the students were able to correctly write the components of vector $\left|\chi_{1}\right\rangle=a|i\rangle+b|j\rangle+$ $c|k\rangle$ as $a=\left\langle i \mid \chi_{1}\right\rangle, b=\left\langle j \mid \chi_{1}\right\rangle$, and $c=\left\langle k \mid \chi_{1}\right\rangle$. Furthermore, Table III shows that on pretest question Q1.a, 26\% of the students simply rewrote the vector $\left|\chi_{1}\right\rangle=a|i\rangle+b|j\rangle+$ $c|k\rangle$ (which was given in the problem statement) as their response.

On the other hand, on pretest question Q1.b, students performed better. Table II shows that $88 \%$ of the students correctly wrote $\left|\chi_{1}\right\rangle \doteq\left(\begin{array}{l}a \\ b \\ c\end{array}\right)$ in the basis $|i\rangle,|j\rangle$, and $|k\rangle$. The dichotomy in students' performance on Q1.a and Q1.b indicates that while students are familiar with representing vectors in matrix form, they do not understand conceptually that $\left|\chi_{1}\right\rangle=a|i\rangle+b|j\rangle+c|k\rangle \doteq\left(\begin{array}{l}a \\ b \\ c\end{array}\right)$ and that to find the component $a$, one can take the inner product, i.e., $\left\langle i \mid \chi_{1}\right\rangle \doteq$ (1 $\left.\begin{array}{lll}1 & 0 & 0\end{array}\right)\left(\begin{array}{l}a \\ b \\ c\end{array}\right)=a$. Tables II and III show that, after working through the QuILT, the difficulties on questions Q1.a and Q1.b were reduced and students performed better.

Incorrectly writing ket vectors as row vectors and bra vectors as column vectors. Table II shows that, on pretest question Q2.a, only $49 \%$ of the students wrote the correct expression for the outer product in the given basis, i.e., $\left|\chi_{1}\right\rangle\left\langle\chi_{2}\right| \doteq\left(\begin{array}{l}a \\ b \\ c\end{array}\right)\left(\begin{array}{lll}d^{*} & e^{*} & f^{*}\end{array}\right)=\left(\begin{array}{l}a d^{*} a e^{*} a f^{*} \\ b d^{*} b e^{*} b f^{*} \\ c d^{*} c e^{*} c f^{*}\end{array}\right)$. Students were not penalized if they did not use the complex conjugate of the components of the vector $\left\langle\chi_{2}\right|$. Furthermore, Table III shows that, on the pretest question Q2.a, 9\% of the students wrote the correct expression for the outer product, i.e., $\left|\chi_{1}\right\rangle\left\langle\chi_{2}\right|$ but
Table II. Percentages of students correctly answering questions on the pretest (pre) and posttest (post) questions.

\begin{tabular}{|l|l|l|l|l|l|l|}
\hline & Q1.a & Q1.b & Q2.a & Q2.b & Q3 & Q4 \\
\hline pre & $49 \%$ & $88 \%$ & $49 \%$ & $63 \%$ & $56 \%$ & $44 \%$ \\
\hline post & $93 \%$ & $95 \%$ & $80 \%$ & $93 \%$ & $93 \%$ & $94 \%$ \\
\hline
\end{tabular}

Table III. Percentages of students displaying difficulties on the pretest (pre) and posttest (post) questions (Q).

\begin{tabular}{|l|l|l|l|}
\hline Q & Difficulty & pre & post \\
\hline Q1.a & $\begin{array}{l}\text { Incorrectly writing the components of a three } \\
\text { dimensional vector in the given basis as } \\
\left|\chi_{1}\right\rangle=a|i\rangle+b|j\rangle+c|k\rangle \text { (which was given } \\
\text { in the problem statement) }\end{array}$ & $2 \%$ \\
\hline Q2.a & $\begin{array}{l}\text { Incorrectly writing matrix forms of bra and ket } \\
\text { vectors, e.g., writing ket vectors as row } \\
\text { matrices and bra vectors as column matrices }\end{array}$ & $2 \%$ \\
\hline Q2.a & $\begin{array}{l}\text { Incorrectly writing the outer product of ket } \\
\text { vector }\left|\chi_{1}\right\rangle \text { with bra vector }\left\langle\chi_{2}\right| \text { as }\left\langle\chi_{2} \mid \chi_{1}\right\rangle .\end{array}$ & $5 \%$ \\
\hline Q2.b & $\begin{array}{l}\text { Incorrectly describing the outer product as a } \\
\text { scalar }\end{array}$ & $19 \%$ & $7 \%$ \\
\hline Q2.b & $\begin{array}{l}\text { Incorrectly describing the outer product as a } \\
\text { column vector }\end{array}$ & $12 \%$ & $0 \%$ \\
\hline Q3 & $\begin{array}{l}\text { Correctly representing the identity operator in } \\
\text { matrix form, i.e., } \hat{I} \doteq\left(\begin{array}{l}100 \\
010 \\
001\end{array}\right) \text { but not in Dirac } \\
\text { notation, i.e., } \hat{I}=|i\rangle\langle i|+| j\rangle\langle j|+| k\rangle\langle k|\end{array}$ & $5 \%$ \\
\hline Q3 & $\begin{array}{l}\text { Writing an incorrect expression for the identity } \\
\text { operator, } \hat{I}=|i\rangle+|j\rangle+|k\rangle\end{array}$ & $14 \%$ & $4 \%$ \\
\hline Q3 & $\begin{array}{l}\text { Writing an incorrect expression for the identity } \\
\text { operator, } \hat{I}=\langle i \mid i\rangle+\langle j \mid j\rangle+\langle k \mid k\rangle\end{array}$ & $5 \%$ \\
\hline Q4 & $\begin{array}{l}\text { Incorrectly writing that the projection operator } \\
\text { that projects vector }\left|\chi_{1}\right\rangle \text { along the direction of } \\
\text { the unit vector }|i\rangle \text { is }\left\langle i \mid \chi_{1}\right\rangle\end{array}$ & $25 \%$ & $5 \%$ \\
\hline
\end{tabular}

wrote incorrect matrix forms of the bra and ket vectors. For example, one student incorrectly wrote the outer product as $\left|\chi_{1}\right\rangle\left\langle\chi_{2}\right|=\left(\begin{array}{lll}a^{*} & b^{*} & c^{*}\end{array}\right)\left(\begin{array}{l}d \\ e \\ f\end{array}\right)$. Other mistakes included $\left|\chi_{1}\right\rangle\left\langle\chi_{2}\right|=$ $(a|i\rangle+b|j\rangle+c|k\rangle)\left(\begin{array}{c}d^{*}|i\rangle \\ e^{*}|j\rangle \\ f^{*}|k\rangle\end{array}\right)$ or $\left|\chi_{1}\right\rangle\left\langle\chi_{2}\right|=\left(\begin{array}{l}a \\ b \\ c\end{array}\right)\left(\begin{array}{l}d \\ e \\ f\end{array}\right)$. These types of responses shed light on students' difficulties with how ket vectors can be represented as column matrices and bra vectors can be represented as row matrices. Tables II and III show that after working through the QuILT, student difficulties on question Q2.a were reduced.

Confusing the inner product with the outer product in Dirac notation. Table III shows that, on pretest question Q2.a, $19 \%$ of the students incorrectly wrote the outer product of ket vector $\left|\chi_{1}\right\rangle$ with bra vector $\left\langle\chi_{2}\right|$ as $\left\langle\chi_{2} \mid \chi_{1}\right\rangle$. Their final answers were typically of the form $\left\langle\chi_{2} \mid \chi_{1}\right\rangle=\left(\langle i| d^{*}+\right.$ $\left\langle j\left|e^{*}+\langle k| f^{*}\right)(a|i\rangle+b|j\rangle+c|k\rangle)=d^{*} a+e^{*} b+f^{*} c\right.$.

Responses of this type indicate that some students had difficulty distinguishing between the concepts of inner product and outer product. Tables II and III show that after 
working through the QuILT, student difficulties on question Q2.a were reduced and students performed better.

Incorrectly describing the outer product as a scalar or a column vector. Students also had difficulty with describing whether or not the outer product is a scalar, column vector, row vector, or a $3 \times 3$ matrix. Table II shows that, on pretest question Q2.b, 63\% of the students correctly stated that the outer product is a $3 \times 3$ matrix. However, Table III shows that, on pretest question Q2.b, 19\% of the students incorrectly claimed that the outer product is a scalar. Students who claimed that the outer product is a scalar usually had written the outer product of ket vector $\left|\chi_{1}\right\rangle$ with bra vector $\left\langle\chi_{2}\right|$ as an inner product in question Q2.a, e.g., $\left\langle\chi_{2} \mid \chi_{1}\right\rangle$. Table III also shows that, on the pretest, $12 \%$ of the students incorrectly claimed that the outer product is a column vector. Students who claimed that the outer product is a column vector sometimes wrote, e.g., $\left|\chi_{1}\right\rangle\left\langle\chi_{2}|=a d| i\right\rangle+$ $b e|j\rangle+c f|k\rangle=\left(\begin{array}{l}a d \\ b e \\ c f\end{array}\right)$. Some students attempted to find the cross product of $\left|\chi_{1}\right\rangle$ with $\left|\chi_{2}\right\rangle$, e.g., $\left|\chi_{1}\right\rangle\left\langle\chi_{2}|=| \begin{array}{lll}i & j & k \\ a & b & c \\ d & e & f\end{array}\right|=$ $(b f-c e)|i\rangle+(c d-a f)|j\rangle+(a e-b d)|k\rangle$ and then claimed that the outer product is a column matrix. Tables II and III show that, after working through the QuILT, student difficulties on question Q2.b were reduced.

Difficulty with the identity operator. Table II shows that, on pretest question Q3, 56\% of the students correctly wrote that the identity operator is $\hat{I}=|i\rangle\langle i|+| j\rangle\langle j|+| k\rangle\langle k|$. Table III shows that, on the pretest question Q3, 16\% of the students correctly wrote the identity operator in matrix form,

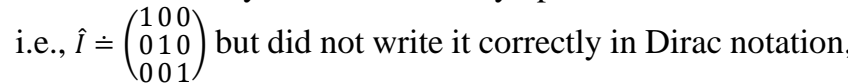
i.e., $\hat{I}=|i\rangle\langle i|+| j\rangle\langle j|+| k\rangle\langle k|$ (the students were asked to write the identity operator in terms of $|i\rangle,|j\rangle$, and $|k\rangle)$. Interviews suggest that some students are more familiar with the identity operator in matrix form and have difficulty with it in Dirac notation. Furthermore, Table III shows that, on the pretest question $\mathrm{Q} 3,14 \%$ of the students wrote $\hat{I}=|i\rangle+|j\rangle+|k\rangle$, indicating that they were confusing ket vectors (column matrices) and operators $(3 \times 3$ matrices $)$. Table III also shows that, on question Q3, 5\% of the students wrote that the identity operator is $\hat{I}=\langle i \mid i\rangle+\langle j \mid j\rangle+\langle k \mid k\rangle$, which demonstrates that they were unable to differentiate between operators and scalar products (numbers). Tables II and III show that, after working through the QuILT, student difficulties on question Q3 were reduced.

Difficulty with projection operator. Table II shows that, on pretest question Q4, less than half of the students correctly wrote that the projection operator that projects vector $\left|\chi_{1}\right\rangle$ along the direction of the unit vector $|i\rangle$ is $|i\rangle\langle i|$. Also, in response to this question, many students correctly stated that a projection of vector $\left|\chi_{1}\right\rangle$ along the direction of the unit vector $|i\rangle$ would give the component of $\left|\chi_{1}\right\rangle$ along $|i\rangle$. For example, in response to question Q4, many students simply wrote the expression $\left\langle i \mid \chi_{1}\right\rangle=a$, which is a correct statement but it is not the answer to the question asked. Table III shows that, on pretest question Q4, 25\% of the students wrote that the projection operator that projects vector $\left|\chi_{1}\right\rangle$ along the direction of the unit vector $|i\rangle$ is $\left\langle i \mid \chi_{1}\right\rangle$. Interviews with some of the students suggest that students with this type of response often did not realize that the projection operator returns both the component and the direction of the projection of state vector $\left|\chi_{1}\right\rangle$ along the unit vector $|i\rangle$, i.e., $|i\rangle\left\langle i \mid \chi_{1}\right\rangle=a|i\rangle$. Examples of incorrect student responses include, "we have found how much of $\left|\chi_{1}\right\rangle$ goes in the direction of $|i\rangle$ " and "the projection operator returns the coefficient for $|i\rangle$." These types of responses indicate that students recognized that the projection operator involves the component of the vector $\left|\chi_{1}\right\rangle$ along the basis vector $|i\rangle$, but they did not realize that the projection operator acting on a generic vector returns the component of the generic vector along the basis vector multiplied by the same basis vector, i.e., $|i\rangle\left\langle i \mid \chi_{1}\right\rangle=a|i\rangle$ (and in general, an operator acting on a vector returns a vector). Tables II and III show that, after working through the QuILT, student difficulties with the projection operator on question $\mathrm{Q} 4$ were greatly reduced.

\section{SUMMARY}

Dirac notation is a compact and elegant notation taught to students in advanced QM courses. Similar to Feynman diagrams in quantum field theory, Dirac notation can simplify the process of solving QM problems. However, this notation is only useful if students develop facility with this notation and do not have cognitive overload [39] when using it. Unfortunately, many advanced students find it challenging to master this notation and have difficulty in translating between the Dirac notation and other representations of the same quantum mechanical entity in different situations. For example, translating between the Dirac notation and matrix representation of the quantum states and operators in a particular basis is often necessary, but challenging. One central reason students struggle with the Dirac notation is that they are unfamiliar with the notation, which is used almost exclusively in the context of QM and not in other physics contexts even though they have learned about vectors and vector spaces. The QuILT strives to help students build a coherent understanding of Dirac notation in threedimensional Hilbert space before learning about quantum concepts using Dirac notation in the context of an $N$ dimensional Hilbert space. We investigated upper-level undergraduate students' difficulties with Dirac notation after traditional lecture-based instruction and used research as a guide to develop, validate and evaluate a QuILT that uses analogical reasoning with the familiar three-dimensional vector space to help students learn about Dirac notation. The in-class evaluation in the undergraduate QM course shows that the student difficulties were reduced significantly after engaging with the QuILT.

\section{ACKNOLWEDGEMENTS}

We thank NSF for award PHY-1806691. 
[1] C. Singh, Student understanding of quantum mechanics, Am. J. Phys. 69, 885 (2001).

[2] D. Zollman, N. S. Rebello and K. Hogg, Quantum mechanics for everyone: Hands-on activities integrated with technology, Am. J. Phys. 70, 252 (2002).

[3] M. Wittmann, R. Steinberg, and E. Redish, Investigating student understanding of quantum physics: Spontaneous models of conductivity, Am. J. Phys. 70, 218 (2002).

[4] G. Zhu and C. Singh, Improving students' understanding of quantum mechanics via the Stern-Gerlach experiment, Am. J. Phys. 79, 499 (2011).

[5] E. Gire and C. Manogue, in Proceedings of the Physics Education Research Conference, Omaha, NE, edited by C. Singh, N. S. Rebello, and P. Engelhardt (AIP, Melville, NY, 2012).

[6] A. Kohnle, I. Bozhinova, D. Browne, M. Everitt, A. Fomins, P. Kok, G. Kulaitis, M. Prokopas, D. Raine and E. Swinbank, A new introductory quantum mechanics curriculum, Eur. J. Phys. 35, 015001 (2014).

[7] E. Marshman and C. Singh, Framework for understanding student difficulties in quantum mechanics, Phys. Rev. ST Phys. Educ. Res. 11, 020119 (2015).

[8] C. Singh and E. Marshman, Review of student difficulties with quantum mechanics, Phys. Rev. ST Phys. Educ. Res. 11, 020117 (2015).

[9] E. Marshman and C. Singh, Investigating and improving student understanding of quantum mechanical observables and their corresponding operators in Dirac notation, Eur. J. Phys. 39, 015707 (2018).

[10] E. Gire and E. Price, The structural features of algebraic quantum notations, Phys. Rev. ST Phys. Educ. Res. 11, 020109 (2015).

[11] P. Emigh, G. Passante, and P. Shaffer, Student understanding of time dependence in quantum mechanics, Phys. Rev. ST Phys. Educ. Res. 11, 020112 (2015).

[12] R. Sayer, A. Maries and C. Singh, A quantum interactive learning tutorial on the double-slit experiment to improve student understanding of quantum mechanics, Phys. Rev Phys. Educ. Res. 13, 010123 (2017).

[13] V. Dini and D. Hammer, Case study of a successful learner's epistemological framings of quantum mechanics, Phys. Rev. Phys. Educ. Res. 13, 010124 (2017).

[14] C. Keebaugh, E. Marshman and C. Singh, Improving student understanding of corrections to the energy spectrum of the hydrogen atom for the Zeeman effect, Phys. Rev. Phys. Educ. Res. 15, 010113 (2018).

[15] E. Marshman and C. Singh, Interactive tutorial to improve student understanding of single photon experiments involving a Mach-Zehnder Interferometer, Eur. J. Phys. 37, 024001 (2016).

[16] R. Sayer, A. Maries and C. Singh, A quantum interactive learning tutorial on the double-slit experiment to improve student understanding of quantum mechanics, Phys. Rev. Phys. Educ. Res. 13, 010123 (2017).

[17] E. Marshman and C. Singh, Investigating and improving student understanding of the probability distributions for measuring physical observables in quantum mechanics, Eur. J. Phys. 38, 025705 (2017).

[18] E. Marshman and C. Singh, Investigating and improving student understanding of quantum mechanics in the context of single photon interference, Phys. Rev. PER 13, 010117 (2017).

[19] C. Keebaugh, E. Marshman and C. Singh, Investigating and addressing student difficulties with the corrections to the energies of the hydrogen atom for the strong and weak field Zeeman effect, Eur. J. Phys. 39, 045701 (2018).

[20] C. Keebaugh, E. Marshman and C. Singh, Improving student understanding of fine structure corrections to the energy spectrum of the hydrogen atom, Am. J. Phys. 87, 594 (2019).

[21] C. Keebaugh, E. Marshman and C. Singh, Improving student understanding of a system of identical particles with a fixed total energy, Am. J. Phys. 87, 583 (2019).

[22] E. Marshman and C. Singh, Validation and administration of a conceptual survey on the formalism and postulates of quantum mechanics, Phys. Rev. PER 15, 020128 (2019).

[23] S. Devore and C. Singh, An interactive learning tutorial on quantum key distribution, Phys. Rev. PER 16, 010126 (2020).

[24] M. Bassok and K. Holyoak, Interdomain transfer between isomorphic topics in algebra and physics, J. Exp. Psychol. Learn. Mem. Cogn. 15, 153 (1989).

[25] N. S. Rebello and D. A. Zollman, Transfer of learning from a modern multidisciplinary perspective, dynamic transfer: A perspective from physics education research in Transfer of Learning from a Modern Multidisciplinary Perspective, edited by J. P. Mestre (Information Age Publishing, Greenwich, CT, 2005), pp. 155-215.

[26] C. Singh, Assessing student expertise in introductory physics with isomorphic problems. I. Performance on nonintuitive problem pair from introductory physics, Phys. Rev. ST Phys. Educ. Res. 4, 010104, (2008).

[27] S. Y. Lin and C. Singh, Using isomorphic problems to learn introductory physics, Phys. Rev. ST Phys. Educ. Res., 7, 020104 (2011).

[28] S. Y. Lin and C. Singh, Using an isomorphic problem pair to learn introductory physics: Transferring from a two-step problem to a three-step problem, Phys. Rev. ST Phys. Educ. Res., 9, 020114, (2013).

[29] J. Mestre, Probing adults' conceptual understanding and transfer of learning via problem posing, J. Appl. Dev. Psychol. 23, 9 (2002).

[30] J. Mestre, Implications of research on learning for the education of prospective science and physics teachers, Phys. Educ. 36, 44 (2001).

[31] K. E. Gray and N.S. Rebello, Transfer between paired problems in an interview, AIP Conf. Proc. 790, 157 (2005).

[32] R. J. Sternberg, Component processes in analogical reasoning, Psych. Rev. 84, 353 (1977).

[33] M. Gick and K. Holyoak, Schema induction and analogical transfer, Cog. Psych. 15, 1 (1983).

[34] K. Holyoak, The Psychology of Learning and Motivation edited by G. Bower (Academic, New York, 1985), Vol. 19.

[35] D. Genter and C. Toupin, Systematicity and surface similarity in the development of analogy, Cog. Sci. 10, 277 (1986).

[36] L. Novick, Analogical transfer, problem similarity and expertise, J. Exp. Psych. Learn. Mem. Cog. 14, 510 (1988).

[37] D. Schwartz, J. Bransford, and D. Sears, Transfer of Learning: Research and Perspectives, edited by J. Mestre (Information Age Publishing, Greenwich, CT, 2005), pp. 1-51.

[38] A Guide to Task Analysis, edited by B. Kirwan and L. Ainsworth, (CRC Press, London, 1992).

[39] J. Sweller, Cognitive load during problem solving: Effects on learning, Cognitive Science 12, 257 (1988). 